\title{
Development and evaluation of surface electrocardiogram in the septal accessory pathway localization in typical Wolff-Parkinson-White syndrome
}

\author{
Si Chu Dung ${ }^{1,2, *}$, Khanh Pham Quoc ${ }^{1,2}$, Dong Tran Van²
}

${ }^{1}$ School of Medicine and Pharmacy, Vietnam National University, Hanoi, Viet Nam

${ }^{2}$ Vietnam Heart Institute, Bachmai Hospital, Hanoi, Viet Nam

\section{Correspondence}

Si Chu Dung, School of Medicine and Pharmacy, Vietnam National University, Hanoi, Viet Nam

Vietnam Heart Institute, Bachmai Hospital, Hanoi, Viet Nam

Email: chudungsi@gmail.com

History

- Received: 13 July 2018

- Accepted: 17 September 2018

- Published: 29 September 2018

DOI :

https://doi.org/10.15419/bmrat.v5i9.479

\section{Check for updates}

\section{Copyright}

( ) Biomedpress. This is an openaccess article distributed under the terms of the Creative Commons Attribution 4.0 International license.

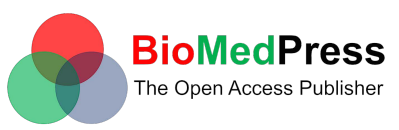

\begin{abstract}
Objectives: This study was designed to characterize the surface electrocardiogram (ECG) of the typical Wolff-Parkinson-White (WPW) syndrome to develop a new algorithm ECG to localize the septal accessory pathways (APs) and to prospectively test the accuracy of the algorithm. Methods: We studied 106 patients, in which 65 patients with typical WPW syndrome who had a single antero-grade with the localization of APs identified by successful radiofrequency catheter ablation (RFCA) to develop a new ECG algorithm for the septal AP localization. Then, this algorithm was tested prospectively in 41 patients to compare to the localization of APs by successful ablation by RFCA (gold standard). Results: In 65 patients with typical WPW syndrome, we found that the 12-lead ECG parameters such as the transition of the QRS complex, delta wave polarity in V1 lead, delta wave polarity in at least 2/3 inferior leads and "QRS pattern" in inferior leads can predict the localization of septal APs with the accuracy ranging from $83.3 \%$ to 100\%. Then, 41 patients were prospectively evaluated by the new derived algorithm to localize the septal APs with high sensitivity and specificity from $84.6 \%$ to $100 \%$. Conclusion: 12-lead ECG parameters in typical WPW syndrome are strongly correlated to the septal AP localization, which can be used to develop a new ECG algorithm to localize septal APs with high accuracy.
\end{abstract}

Key words: 12-lead ECG, Accessory pathway localization, Septal AP, WPW syndrome

\section{INTRODUCTION}

Wolff-Parkinson-White (WPW) syndrome associated with an accessory AV connection (called Kent Bundle). The 12-lead ECG is characterized by a shortened PR, prolonged QRS, with Delta wave ${ }^{1,2}$.

Nowadays, radiofrequency catheter ablation (RFCA) of the accessory pathway (AP) is required to precisely localize the APs along the mitral and tricuspid annulus (gold standard) ${ }^{2}$. Still, 12-lead ECG is the first step to localize AP in patients with WPW syndrome. The data obtained from the ECG parameters can be helpful in planning and shortening the RFCA and X-ray procedures $^{2}$.

Some ECG based algorithms have been published to predict the location of the septal $\mathrm{APs}^{1-4}$. However, many studies showed that it was more difficult to ablate in this area compared to other locations, because the septal area is a complex anatomic structure where four cardiac chambers and the coronary sinus come in close proximity. Furthermore, antero-septal and midseptal regions are close to His bundle and AV node ${ }^{5}$. Therefore, the purpose of this study was to analyze the 12-lead ECG of septal APs successfully localized by RFCA to develop a new ECG algorithm using simple parameters and to test this algorithm to predict APs location prospectively.

\section{METHODS}

\section{Study design}

Observational, cross-sectional, retrospective and prospective study. Select to specify make the standard for the study.

\section{Patient inclusion and exclusion criteria}

\section{Patient inclusion criteria}

Patients included in the study must have typical WPW syndrome and have a single antero-grade AP which was successfully localized by RFCA.

\section{Patient exclusion criteria}

Patients excluded from the study had one of the following criteria: not treated with RFCA, not having enough information during their treatment, not agreeing to participate in the study, not having successful RFCA, or having more than one AP. 


\section{Study contents}

We studied 106 patients with typical WPW syndrome who have a single septal AP from January 2001 to May 2017 at Vietnam Heart Institute, Bach Mai Hospital. There are two phases of the study:

Phase 1 (from January 2001 to June 2016): 65 patients with typical WPW syndrome who had a single antero-grade septal AP identified by successful RFCA were enrolled to develop a new ECG algorithm to localize septal APs using simple parameters.

Phase 2 (from June 2016 to May 2017): Then, this algorithm was prospectively tested in 41 patients to compare to the location of septal APs successfully ablated by RFCA from June 2016 to May 2017.

Using 12-lead ECG, WPW syndrome is characterized by a shortened PR interval $<120$ milliseconds, prolonged QRS duration $\geq 110$ milliseconds, with a delta wave, secondary ST and T wave changes which are directly opposite to the significant Delta wave and QRS vector ${ }^{1}$.

The annulus position of septal APs has four regions (antero-septal, mid-septal, left or right posteroseptal). RFCA for APs is conducted through the ablating action of the catheter tip at the intersection of the AP and mitral valve or tricuspid valve. Localization of APs is confirmed by the catheter position of successful RFCA of each location (gold standard) ${ }^{2}$.

The medical treatment was conducted in the catheterization laboratory at Vietnam National Heart Institute, Bach Mai General Hospital, Hanoi, Vietnam. We performed a full spectrum of cardiac and vascular interventions with the Allura Xper FD20 X-ray biplane system of Philip (Holland), programmed cardiac stimulator instrument (integrated in the cardiac stimulator EP-4 system), Cardiac stimulator EP-4 system, Radio Anpere Genetator instrument, diagnostic EP catheter, mapping and ablation catheter by St. Jude (American).

\section{Statistical Analysis}

We confirmed APs localization by successful RFCA as the dependent variable and the 12-lead ECG parameters as the independent variables. Value of $\mathrm{p}<$ 0.05 was considered statistically significant. Calculated values include sensitivity (Se), specificity (Sp), positive predictive value (PPV), and negative predictive value (NPV) of the predicted AP locations. IBM SPSS 21.0 software was used for data analysis.

\section{Research ethics}

The research proposal, database, medical records, and results were approved by the committee of science at the Vietnam University of Military Medicine.
The RFCA used in the research has been demonstrated to be highly effective and safe. The indication and safety of the medical treatment were approved by the hospital ethics committees at Bach Mai hospital.

The patients were explained about the procedure, and they signed the consent for ablation catheter. All patient's medical records were kept confidential and used for research purpose only.

\section{RESULTS}

The study population consists of 106 patients, 53 men (50\%) and 53 female (50\%), with age of $43.7 \pm 14.9$ years (from 18 to 80 years of age).

\section{Characteristic of 12-lead ECG to localize septal accessory pathways}

The study population consists of 65 patients (Group I), 35 males (53.8\%) and 30 females (46.2\%), with age of $42.1 \pm 14.8$ years (from 19 to 80 years of age).

Septal APs were found in 65 patients, in which 29 patients had right postero-septal APs(44.6\%), 25 patients had left postero-septal APs (38.5\%), 6 patients had mid-septal APs (9.2\%), 5 patients had anteroseptal APs (7.7\%).

\section{Transition characteristics of the QRS complex on 12-lead ECG with septal location}

The most common classified transition of septal APs location was at V1, V2 lead (between V2V3 including) found in 58 patients, which is $89.2 \%$ of the total number of septal APs. 38 patients had right posteroseptal, which is $95 \%$ of the total number of right septal APs (Antero-septal had 4/5 patients, mid-septal had 5/6 patients, right postero-septal had 27/29 patients), and left postero-septal had $22 / 25$ patients (88\%).

\section{Characterization of delta wave polarity in the $V 1$ lead with left or right septal}

Left septal APs commonly had a positive delta wave V1 lead, which was found in 23/25 patients (92\%). Besides, right septal APs commonly had a negative delta wave V1 lead, which was found in $35 / 40$ patients (87.5\%).

Left septal APs commonly had a positive QRS complex V1 lead, which was found in 11/25 patients (44\%). Also, right septal APs usually had a negative QRS complex at the V1 lead, which was found in $37 / 40$ patients $(92.5 \%)$. 

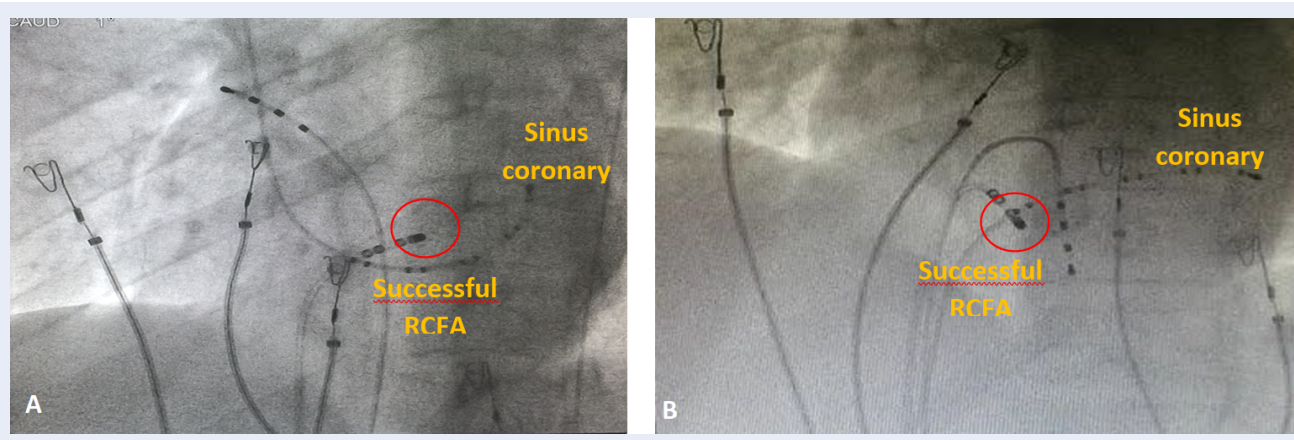

Figure 1: Catheter position for radiofrequency ablation of accessory pathway. A: Catheter position of successful RFCA at the mid-septal (Red circle), $30^{\circ}$ LAO. B: Catheter position of successful RFCA at the right posteroseptal (Red circle), $30^{\circ}$ LAO.

\section{Characterization of delta wave polarity in at least 2/3 inferior lead with antero-septal or postero-septal APs}

Antero-septal APs commonly had a positive delta wave at least $2 / 3$ inferior lead, which was found in $5 / 5$ patients (100\%). Also, postero-septal APs usually had a negative delta wave at least $2 / 3$ inferior lead, which was found in $50 / 54$ patients $(92.6 \%)$

Classified morphology QRS complex (Qrs, qRs, qrS) of mid-septal location was most common at the inferior lead, which was found in 5/6 patients (83.3\%). Among 59 non-mid-septal Aps, (antero-septal and postero-septal), only $8 / 59$ patients (13.6\%) had a morphology QRS complex as described above.

The accuracy of the new ECG algorithm for localizing accessory pathways

The study population consists of 41 patients (Group II), 18 males (43.9\%) and 23 females (56.1\%), with age of $46.3 \pm 14.9$ years (from 18 to 73 years of age).

Septal APs were found in 41 patient with 23 patients had right postero-septal APs (21.1\%), 13 patients had left postero-septal APs (11.9\%), four patients had mid-septal APs (3.7\%), and one patient had antero-septal APs (0.9\%).

Localization of septal or no septal locations accessory pathways group by transition characteristics of the QRS complex on 12-lead ECG

The accuracy of the algorithm in localizing septal or non-septal APs using transition characteristics of the QRS complex at V1V2 or after V1V2 was significantly high, giving the sensitivity of $87.8 \%$ (for the right septal's $89.9 \%$ and left septal's $84.6 \%$ ).

\section{Localization of left or right septal accessory pathways by delta wave polarity in $\mathrm{V} 1$ lead}

The accuracy of the algorithm in localizing left-side or right-side septal APs by a positive or negative delta wave V1 was significantly high, giving the sensitivity of $100 \%$, specificity of $89.3 \%$, PPV of $81.3 \%$ and NPV of $100 \%$.

Localization of antero-septal or posteroseptal accessory pathways group by the delta wave polarity in at least $2 / 3$ inferior lead on 12-lead ECG

The accuracy of the algorithm in localizing anteroseptal or postero-septal APs by a positive or negative delta wave at V1 was significantly high, giving the sensitivity of $100 \%$, specificity of $88.9 \%$, PPV of $20 \%$ and NPV of $100 \%$.

The accuracy of the algorithm in localizing mid-septal or non-mid-septal (antero-septal and postero-septal) APs by Qrs pattern (Qrs, qRs, qrS) or no Qrs pattern at inferior leads was significantly high, giving a sensitivity of $75 \%$, specificity of $91.9 \%$, PPV of $50 \%$ and NPV of $97.1 \%$.

Overall, the accuracy of the new ECG algorithm in localizing APs sites was high according to Tables 1, 2, 3 and 4 .

All patients $(n=41)$ with septal APs required less procedure time with the mean value of $54.0 \pm 24.3 \mathrm{~min}$ utes (ranging from 15 to 180 minutes) and less fluoroscopy time with the mean value of $7.4 \pm 5.0$ minutes (ranging from 2 to 20 minutes). 
Table 1: Transition characteristics of the QRS complex with septal locations

\begin{tabular}{cccc}
\hline $\begin{array}{c}\text { Location of AP } \\
\text { Position of the transition zone }\end{array}$ & Right septal & Left septal & $\begin{array}{c}\text { Total (septal } \\
\text { location) }\end{array}$ \\
\hline V1V2/V2-V3 & 25 & 11 & 36 \\
After V1V2 ( V3-V6) & 3 & 2 & 5 \\
Total (n) & 28 & 13 & 41 \\
\hline
\end{tabular}

Table 2: Delta wave positive/negative in V1 with left/right septal

\begin{tabular}{cccc}
\hline $\begin{array}{c}\text { Location } \\
\text { Delta wave polarity at V1 }\end{array}$ & Left septal & Right septal & Total \\
\hline Positive delta wave at V1 & $13(100 \%)$ & $3(10.7 \%)$ & 16 \\
Negative delta wave at V1 & $0(0 \%)$ & $25(89.3 \%)$ & 25 \\
Total (n) & 13 & 28 & 41 \\
\hline
\end{tabular}

Table 3: Delta wave polarity in at least 2/3 inferior lead (DII, DIII, aVF)

\begin{tabular}{cccc}
\hline \hline $\begin{array}{c}\text { Location } \\
\text { Delta wave polarity in inferior lead }\end{array}$ & Antero-septal & Postero-septal & Total \\
\hline Positive delta wave in at least 2/3 inferior & 1 & 4 & 5 \\
Negative delta wave in at least 2/3 inferior & 0 & 32 & 32 \\
Total (n) & 1 & 36 & 37 \\
\hline
\end{tabular}

Table 4: Morphology QRS complex in the inferior lead with septal

\begin{tabular}{cccc}
\hline $\begin{array}{c}\text { Location } \\
\text { QRS complex morphology }\end{array}$ & Mid-septal & $\begin{array}{c}\text { No mid-septal (Antero-septal and } \\
\text { postero-septal) }\end{array}$ & Total \\
\hline $\begin{array}{c}\text { Qrs pattern (Qrs, qRs, qrS) } \\
\text { No Qrs pattern (Qrs, qRs, }\end{array}$ & 3 & 3 & 6 \\
qrS) & 1 & 34 & 35 \\
Total (n) & 4 & 37 & 41 \\
\hline
\end{tabular}

Table 5: Sensitivity, Specificity, PPV and NPV Value of the Proposed Algorithm in localizing APs in 41 patients

\begin{tabular}{ccccc}
\hline Septal Accessory Pathway Site $(\mathbf{n}=\mathbf{4 1})$ & Se (\%) & Sp (\%) & PPV (\%) & NPV (\%) \\
\hline Sensitivity for accuracy for the Septal APs & $87.8 \%$ & & & \\
Left or right septal accessory pathways & $98.3 \%$ & $89.3 \%$ & $81.3 \%$ & $97.9 \%$ \\
Antero-septal or postero-septal accessory pathways & $100 \%$ & $88.9 \%$ & $20 \%$ & $100 \%$ \\
$\quad$ Mid-septal or no mid-septal accessory pathways & $75 \%$ & $91.9 \%$ & $50 \%$ & $97.1 \%$ \\
$\begin{array}{c}\text { Mid-septal or right accessory pathways (antero-septal and } \\
\text { right postero-septal) }\end{array}$ & $75 \%$ & $92.3 \%$ & $75 \%$ & $92.3 \%$ \\
\hline
\end{tabular}




\section{DISCUSSION}

\section{Characterization}

Transition characteristics of the QRS complex on 12-lead ECG with septal location

Classified transition of the septal location was most common at V1, V2 lead (87.8\%). The septal APs location with anatomic relation to the atrioventricular junction in relation to other cardiac structures, especially in the septal region's transitional cell zone ${ }^{5}$.

Some algorithms based on ECG have not been able to distinguish between antero-septal and right anterolateral APs, and between postero-septal and posterolateral (left or right). However, many studies showed that the transition of QRS complex could be used to predict locations of septal or free wall $\mathrm{APs}^{1-4}$.

\section{Characterization of delta wave polarity in the $\mathrm{V} 1$ lead with left or right septal}

Left septal APs commonly have a positive delta wave at V1 lead (100\%), while right septal APs usually have a negative delta wave at V1 lead (89.3\%). This is very useful to select an appropriate catheter approach such as in the vein or artery. Currently, all right septal APs (right-sided APs) are ablated using trans-venous atrial approach through the femoral vein, while left septal pathways (left-side APs) are ablated using retrograde arterial approach. If this approach fails, the pathway is ablated using antegrade transeptal approach ${ }^{2}$.

Some ECG algorithms have been published to predict the location of left-sided or right-sided APs by positive or negative delta wave ${ }^{1-4}$. Besides, some other studies also diagnosed left or right-side APs by other ECG parameters such as $\mathrm{R} / \mathrm{S}$ ratio $<0.5$ or $\mathrm{R} / \mathrm{S}>0.5$ in V1 lead ${ }^{6}$, positive or negative QRS complex in V1 to diagnose left or right-side $\mathrm{APs}^{7}$.

Characterization of delta wave polarity in at least 2/3 inferior lead (DII, DIII, AVF) with antero-septal or postero-septal

Using characteristic electrocardiogram, we observed strong positive delta waves in at least $2 / 3$ inferior leads (II, III, aVF) in a patient with an antero-septal (100\%). On the other hand, we observed a robust negative delta wave in at least $2 / 3$ inferior leads (II, III, aVF) in patients with postero-septal APs (88.9\%).

Successful ablation was performed at a location on the antero-septal annulus where a His bundle electrogram being recorded. The ablation was very anterior, thus was not in the vicinity of the compact AV node ${ }^{3}$.

The posterior region is a triangular structure between the left ventricle and the right ventricle, located at the base of the Koch's triangle, which can be mixed between left and right in pre-stimulus levels. The postero-septal region is located explicitly between the postero-superior process of the left ventricle and inferior wall or the right atrium. Many studies have shown that it is difficult in discriminating left or rightsided postero-septal APs; however, it has been suggested that a positive/negative delta wave in V1 can further differentiate between left/right sided posteroseptal APs ${ }^{1,8,9}$.

Some ECG algorithms have been published to predict the location of Antero- or postero- APs by positive or negative delta waves in the inferior lead as below ${ }^{1,3,6}$. However, some studies only focused on certain locations of antero-septal and postero-septal APs ${ }^{6}$.

Many studies have reported that the mid-septal is the only correct muscular septal area between the offset attachments of the mitral and tricuspid valves, while the previously named anterior and posterior septal regions are those regions that are anterior and posterior to the right septum. Mid-septal pathways are inserted into the Koch's triangle region, close to the compact AV node. Accurate assessment of pathway slant and ablation long the ventricular insertion site by using cryo-energy may be required to minimize the risk of AV block when ablating pathways in this region ${ }^{3}$.

D'Avila A $(n=140)$ showed that the polarity of the QRS complex did not correctly localize midsepta1 APs. However, lead DIII showed a very similar QRS complex morphology in $5 / 10$ patients with mid-septal APs. The negative QRS complex in lead DIII was characterized by a large " $Q$ ' wave followed by a small " $r$ " wave and an " $s$ " wave. This "Qrs pattern" in lead DIII was highly specific for midsepta1 APs. However, the sensitivity was not high. Probably, D'Avila A only used tQrs pattern on DIII lead to localize mid-septal $\mathrm{AP}^{7}$.

Classified QRS complex morphology (Qrs, qRs, qrS) of mid-septal locations was commonly found at inferior lead (DII, DIII, aVF), which was found in 5/6 patients with mid-septal APs (83.3\%). This "Qrs pattern" in at least $1 / 3$ inferior lead was able to distinguish mid-septal APs from other septal locations. Among other septal locations (60 patients), only eight patients with postero-septal APs (13.3\%) were found to have this QRS complex morphology.

Besides, 8 out of 59 patients (13.6\%) diagnosed with an abnormal posterior septum had abnormal signs in mid-septal regions indicated by imaging results. That may be because two areas have similar anatomical features. Given the anatomical feature of the right midseptal region, which is located between the anterior septum and posterior septum, forming Koch's triangle, incorrect images can be identified by technicians. 

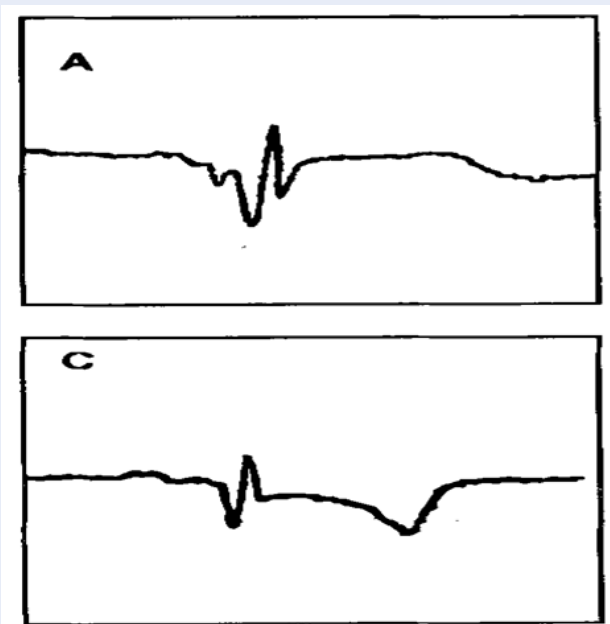

Figure 2: ECG with QRS complex morphology (Qrs) (D'AvilaA.) [7]
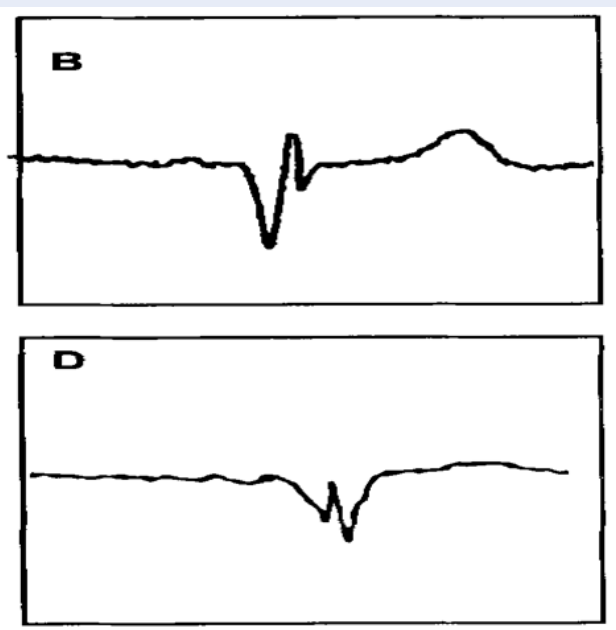

The accuracy of the new ECG algorithm for localizing septal accessory pathways

We developed a new algorithm using some simple ECG parameters such as transition QRS complex at V1V2 or after V1V2 lead to localize septal or lateral APs, positive/negative delta wave at V1 lead to localize left or right side APs, positive/negative delta waves in at least 2/3 inferior to localize Antero- or posteroAPs, and Qrs pattern (QRS, qRs, qrS) in at least $1 / 3$ inferior lead (DII, DIII, aVF) to localize mid-septal APs (Figure 3).

Then, this algorithm was prospectively tested in $41 \mathrm{pa}-$ tients to compare with the locations of APs identified by successful ablation by RF, which was used to calculate sensitivity, specificity, positive predictive value and negative predictive value of the diagnosed algorithm in predicting the locations of APs (Tables 1, 2, 3,4 and 5). For the left or right side APs, by using positive/negative delta waves at V1 lead (Table 1); Chang CE (1995) achieved Se of $94.4 \%$ and Sp of $87.5 \%{ }^{8}$. Similarly, for septal or lateral APs by using transition QRS complex at V1V2 or after V1V2 lead (Table 2); Dar M (2008) achieved Se of $97 \%$ and Sp of $95 \%{ }^{4}$. For the antero or postero APs by positive/negative delta waves in inferior (Table 3), Dar M achieved Se and Sp from 85 to $100 \%{ }^{4}$. This "Qrs pattern" in the inferior lead (DII, DIII, aVF) was highly sensitive (75\%) and specific (91.9\%) for mid-septal locations (Table 4) ${ }^{7}$. There are three out of 36 cases showed specific signals of mid-septal regions (Table 4). However, they were actually in the posterior septum, which can be explained by the fact that these two regions are close to each other, which have same physiological features and some transitional muscles are running through these two regions. Indeed, the results of successful radiofrequency ablation indicated that the three patients had APs which were very close to the mid-septal areas. The diagnostic results confirmed our hypothesis. We suspected the signals are indicating posterior septum localization because there were some signals from posterior regions: a negative delta wave in at least $2 / 3$ inferior lead (DII, DIII, aVF), specifically in the case having a positive delta wave in the V1 lead. Above parameters of 12-lead ECG was able to predict successful ablation sites, which significantly shortened the procedure time and fluoroscopy time, and enhanced the fluoroscopy safety (Table 6) ${ }^{12}$.

The study significantly improved patients' hospital experiences. To be specific, the research helped reduce X-ray exposure to decrease the side-effects for both doctors and patients from radiologic techniques. Also, the study provided a fast method to identify root causes of arrhythmia as well as to localize APs, and to forecast any accident during RFCA, which decreased patients treatment deration, and increased medical staffs' performance.

\section{CONCLUSIONS}

We developed and validated a new algorithm to localize septal APs. We found that the left septal APs mostly have a positive delta wave at V1 lead (92\%) and right septal APs mostly have a negative delta wave V1 lead (87.5\%). The classified transition of septal location is most common at V1, V2 lead (89.2\%). Antero-septal APs mostly have a positive delta wave at least $2 / 3$ inferior lead (100\%), and postero-septal 

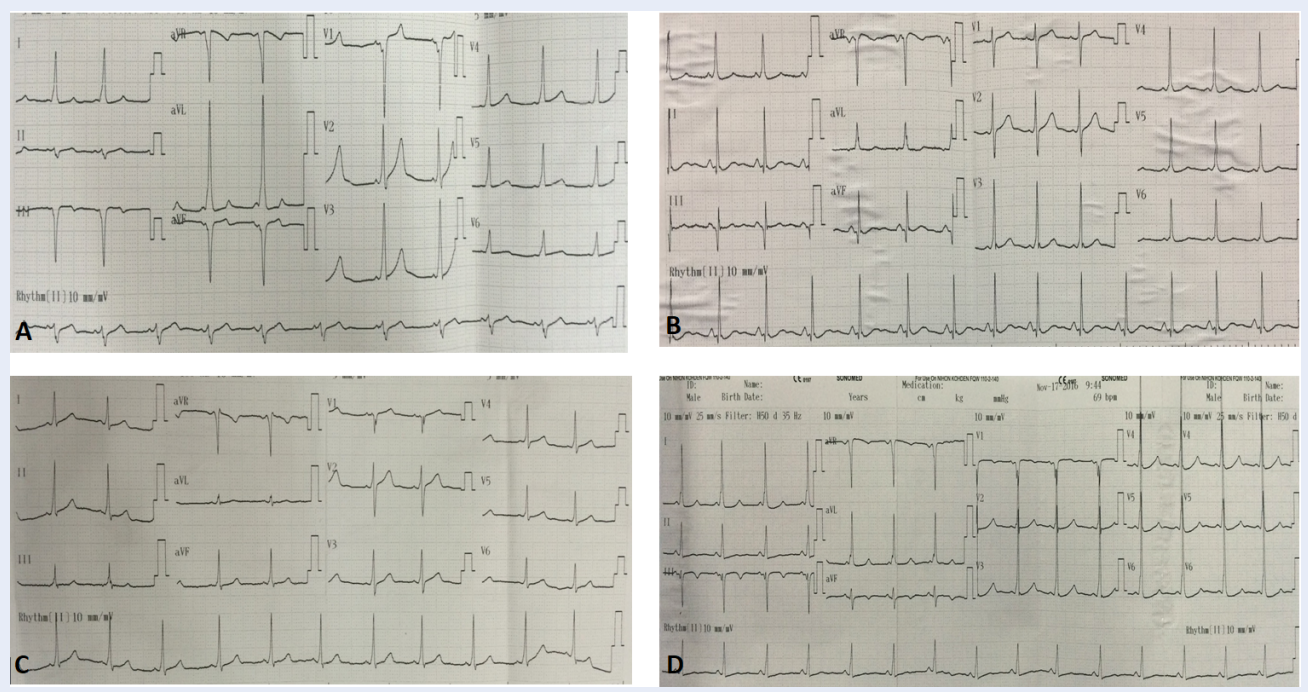

Figure 3: ECG in patient with WPW syndrome. A: Right postero-septal (Negative delta wave in V1, QRS transition in V1, Negative QRS in 2/3 inferior lead (DIII, aVF). B: Left postero-septal (Positive delta wave in V1, QRS transition in V2, Negative QRS in 3/3 inferior lead (DII, DIII, aVF). C: Antero-septal (Negative delta wave in V1, QRS transition in V1, Positive QRS in 3/3 inferior lead (DII, DIII, aVF). D: Right Mid-septal (Negative delta wave in V1, QRS transition in V1, QRS with specify morphology's qrS in DIII).

Table 6: Comparison of procedural parameters in other studies about procedure time and fluoroscopy time

\begin{tabular}{cccc}
\hline Authors & Accessory pathway location & $\begin{array}{c}\text { Mean Procedure } \\
\text { time (minute) }\end{array}$ & $\begin{array}{c}\text { Mean Fluoroscopy time } \\
\text { (minute) }\end{array}$ \\
\hline Calkins H. (1992) & WPW syndrome (n=250) & $134 \pm 75$ & $47 \pm 33$ \\
& Right posteroseptal (n=26) & $99.8 \pm 48.0$ & $23.2 \pm 12.8$ \\
Dong TV. (2006) & $\begin{array}{c}\text { Antero-septal \& mid-septal } \\
(\mathrm{n}=10)\end{array}$ & $106.7 \pm 56.8$ & $22.9 \pm 16.4$ \\
Haghjoo M. $(2008)^{10}$ & Posteroseptal (n=94) & - & $40.0 \pm 13.0$ \\
Our research $(2016-2017)$ & Septal APs (n=41) & $54.0 \pm 24.3$ & $7.4 \pm 5.0$ \\
\hline
\end{tabular}

APs mostly have a negative delta wave at least $2 / 3$ inferior lead (92.6\%). Mid-septal APs commonly have a negative QRS with specific morphology (Qrs, qRs, qrS) at least $1 / 3$ inferior lead (83.3\%).

The new algorithm was proved to be highly accurate and sensitive (from $83.3 \%$ to $100 \%$ ), with high specificity $(75 \%$ to $100 \%)$. The mean procedure time and fluoroscopy were significantly shortened and safer when using the above parameters to predict the location of septal APs.

\section{COMPETING INTERESTS}

The authors declare no potential conflict of interest.

\section{AUTHORS' CONTRIBUTIONS}

Si CD designed the study, collected data, conducted the statistical analysis and wrote the manuscript.
Khanh PQ and Dong TV are revised the manuscript during the review process.

\section{ACKNOWLEDGMENTS}

Vietnam University of Military Medicine \& Vietnam National Heart Institute, Bachmai Hospital.

\section{ABBREVIATIONS}

AP: Accessory pathway

APs: Accessory pathways

AV: Atrial Node

ECG: Electrocardiogram

RFCA: Radiofrequency catheter ablation

WPW: Wolff-Parkinson-White

\section{REFERENCES}

1. Surawicz B, Knilans TK, Chou TC. Chou's Electrocardiography in clinical practice: Adult and Pediatric; 2008. 
2. Basiouny $\mathrm{T}$, Chillou $\mathrm{CD}$, Fareh $\mathrm{S}$. Prospective validation of a sezer ecg algorithm for localization of accrssory pathways in patients with wolff-parkinson-white syndrome. AAMJ. 2012;10:2.

3. Macedo PG, Patel SM, Bisco SE, Asirvatham SJ. Septal accessory pathway: anatomy, causes for difficulty, and an approach to ablation. Indian Pacing and Electrophysiology Journal. 2010;10:292-309. null. Available from: https://www.ncbi. nlm.nih.gov/pmc/articles/PMC2907089/.

4. Dar MA, SHEIKH SH, ABID AR, MALLICK NH. Localization of ACcessory Pathways According to AP Fitzpatrick Ecg Criteria in Patents with Wolff-Parkinson-White Syndrome in Our Population. Pakistan Heart Journal. 2012;41(3-4):21-27.

5. Cheng A, Shah A, Hogue CW. Cardiac Electrophysiology: Diagnosis and Treatment. Anesthesiology. In: Kaplan JA, Reich DL, Konstadt SN, editors. Kaplans Cardiac Anesthesia Expert Consult Premium. Saunders; 2011.

6. Taguchi N, Yoshida N, Inden Y, Yamamoto T, Miyata S, Fujita M. A simple algorithm for localizing accessory pathways in patients with Wolff-Parkinson-White syndrome using only the R/S ratio. Journal of Arrhythmia. 2014;30:439-43. Available from: DOI:10.1016/j.joa.2013.10.006.

7. d'Avila A, Brugada J, Skeberis V, Andries E, Sosa E, Brugada P. $A$ fast and reliable algorithm to localize accessory pathways based on the polarity of the QRS complex on the surface ECG during sinus rhythm. Pacing and Clinical Electrophysiology.
1995;18:1615-27. Available from: DOI:10.1111/j.1540-8159. 1995.tb06983.x.

8. Chiang CE, Chen SA, Teo WS, Tsai DS, Wu TJ, Cheng CC, et al. An accurate stepwise electrocardiographic algorithm for localization of accessory pathways in patients with WolffParkinson-White syndrome from a comprehensive analysis of delta waves and $\mathrm{R} / \mathrm{S}$ ratio during sinus rhythm. The American journal of cardiology. 1995;76(6):40-46. Available from: 10.1016/j.joa.2013.10.006.

9. Calkins H, Langberg J, Sousa J, el Atassi R, Leon A, Kou W. Radiofrequency catheter ablation of accessory atrioventricular connections in 250 patients. Abbreviated therapeutic approach to Wolff-Parkinson-White syndrome. Circulation 1992;85:1337-46. Available from: Doi:10.1161/01.cir.85.4. 1337.

10. Haghjoo M, Mahmoodi E, Fazelifar AF, Alizadeh A, Hashem MJ, Emkanjoo Z. Electrocardiographic and electrophysiologic predictors of successful ablation site in patients with manifest posteroseptal accessory pathway. Pacing and Clinical Electrophysiology. 2008;31:103-11. Available from: DOI:10.1111/ j.1540-8159.2007.00933.x.

11. Dong TV. Research in Electrophysiology and treatment of Wolff-Parkinson-White Syndrome by Radiofrequency Catheter Ablation; 2006

12. Singer I. Clinical manual of electrophysiology. 1993. p. 52-68. 